\title{
Insilico and Invitro Evaluation of Dy(III) Complex on Serum Albumin as well as Amino Acid Loops in M-Protease of SARS-Coronavirus-2
}

\author{
M. Santhiya ${ }^{1} \&$ T. Lurthu Pushparaj ${ }^{2 *}$ \\ ${ }^{I}$ Department of Chemistry, UG, T.D.M.N.S College, Tamil Nadu-627113, India. \\ ${ }^{2 *}$ Assistant Professor, Department of Chemistry, PG, T.D.M.N.S College, Tamil Nadu-627113, India. \\ DOI: http://doi.org/10.38177/ajast.2021.5217
}

Copyright: @2021 M.Santhiya \& T.Lurthu Pushparaj. This is an open access article distributed under the terms of the Creative Commons Attribution License, which permits unrestricted use, distribution, and reproduction in any medium, provided the original author and source are credited.

\section{ABSTRACT}

Countless proteins contain signal patterns that act as entry point and guiding others protein to particular cell frameworks. Likewise, the mutation in the main chain protease of "spike" proteins seemed to be crucial in viral infection and recombination in covid-19. A certain drug that binds to the sequence will further prevent coronavirus synchronization. To address this, we designed and characterized a new complex for Covid-19 inhibition that contains 1,10-phenantroline as a ligand and Dy(III) as more than just a metal.

The absorption character in neat solvent, in the existence of 4.5 percent BSA, as well as the biological interplay of COVID-19 virus via molecula docking, has been carried out to determine the complex's efficacy in this pandemic situation. The complex's absorbance spectra shows intense peaks that correspond to intra ligand $\pi-\pi^{*}, \mathrm{n}-\pi^{*}$, and charge transfer transitions. It also shows a red shift in peaks corresponding to LCT and MLCT transitions in 4.5 percent of BSA. The molecular docking analysis of the complex with the COVID-19 virus (PDB: 6LU7) reveals a strong polar interaction with various amino acids in the spike protein.

The complex has awesome binding energies in the $-8.8 \mathrm{kcal} \mathrm{mol}^{-1}$ range. As a result, they are legit contender compounds for the development of candidates against SARS-COV-2. We also evaluated the complex's basic energies, such as potential and steric energy, using Gaussian energy calculations.

Keywords: Human Covid-19, SARS-Molecular docking, BSA absorption, Dy(III) complex, Phenanthroline drug.

\section{Introduction}

Lanthanide single-molecule complexes are appealing because they have distinct fluorescent properties due to $\mathrm{f}$-f electronic transitions. Luminescent lanthanide-based biochemical materials have sparked widespread professional curiosity due to their numerous applications in different fields such as bio-imaging [1,2], chemical sensing [3,4]. The majority of lanthanides have a half filled submerged 4 f-electron shell just below a filled $6 s 2$ shell. The addition of $4 \mathrm{f}$ electrons with parallel spins results in a large magnetic moment as well as a large total and orbital angular momentum. These "inextinguishable" angular moments produce a diverse atomic and molecular structure as well as collective structure of these systems [4,5]. Lanthanide ions have very different coordination chemistry than transition metal ions. Coordination numbers $(\mathrm{CNs})$ ranging from eight to twelve are common for lanthanide ions due to their large ionic radii. The resulting coordination polyhedrons are square antiprism, bicapped trigonal prism, triangular dodecahedron $(\mathrm{CN}=8)$, tricapped trigonal prism, monocapped square antiprism $(\mathrm{CN}=9)$, bicapped square antiprism, bicapped dodecahedron, tetrakaidecahedron $(\mathrm{CN}=10)$, icosahedron $(\mathrm{CN}=12)$, and others. Lanthanide coordination chemistry has advanced significantly in recent years, but lanthanide species with CN 8 are still uncommon. Dysprosium was discovered in 1886 by Paul Émile Lecoq de Boisbaudran, but it was not isolated in pure form until the 1950s, thanks to the development of ion-exchange techniques. Dyprosium's magnetic properties are caused by its unfilled $4 \mathrm{f}$ shell. Dysprosium is ferromagnetic below $85^{\circ} \mathrm{K}$ at $1 \mathrm{~atm}$, anti-ferromagnetic between $85^{\circ}$ and $178.5^{\circ} \mathrm{K}$, and paramagnetic above $178.5^{\circ} \mathrm{K}$. We previously reported the use of F-block metals with high magnetic moments in the design of MRI contrast enhancing agents and smart multimodal cancer imaging drugs 
where other metals could not be substituted [6-14]. While dysprosium does not currently have a wide range of applications, we chose this high magnetic Dy(III) metal to investigate its binding properties with macromolecules and covid-19 infections. Phenanthroline (phen) is a heterocyclic organic compound that forms strong complexes with most $\mathrm{d}$ and $\mathrm{f}$ block metal ions and is used as a ligand in coordination chemistry [15,16]. 1,10-Phenanthroline is a metallopeptidases inhibitor, with one of the first reported cases in carboxypeptidase A [17]. The enzyme is inhibited by removing and chelating the metal ion required for catalytic activity, resulting in an inactive Apo enzyme. With a much lower affinity for calcium, 1,10-phenanthroline primarily targets zinc metallopeptidases [18]. Bovine serum albumin (BSA) is a protein isolated from cow serum albumin. BSA is used in molecular biology to stabilize some restriction enzymes during DNA digestion. In many applications, BSA is regarded as a universal blocking reagent because it has no effect on the functions of other proteins that do not require it for stabilization. BSA is also widely used to estimate the number of units of other proteins, including the Bradford Protein Assay [19], by likening an unidentified amount of protein to known concentrations of BSA.

Chronic respiratory syndromes during subsequent two major outbreaks of lethal Coronavirus, SARS-CoV-1 in 2003 [20] and Middle-Eastern Respiratory Syndrome (MERS) in 2012 [21], and also the existing SARS-CoV-2 pandemic, frequently resulted from corrupt and inefficient immune responses triggered by the host 's immune platform's interplay with the virus [22,23]. While strong immune responses are required to contain and clear viral infection, excessive inflammation can damage blood vessels, delay tissue healing after viral clearance, and result in acute inflammatory responses and sepsis. The degree and severity of immune-response pathologies differ greatly between individuals in the case of SARS-CoV-2. Because of the complexities of the many patterns of SARS-CoV-2 response, we urgently require methods to identify important biological mechanisms that act at different stages of the infection and enable us to reliably identify differences in path that leads and gene activity between clinical practice, tissues within patients, individuals with pre-existing conditions, and age. The immune system is complex, sensitive, and dynamic, with a delicate balance of triggers, high-gain feed-back loops, and complex interactions among its many agents, exacerbating view of experimental measurements of immune-response components as well as the origins of unique variance. In this case, detailed mathematical models of patient-specific immune responses may help us understand the range of possible immune responses and how they depend on patient-specific variables such as initial exposure level and co-infections, age, sex, pre-existing conditions and medications, and so on, for diagnostic, prognostic, and therapeutic purposes. Furthermore, in severe cases, COVID-19 symptoms may include blood and vascular disruption, implying that the co-activation of other pathways with detrimental effects may play a role in disease outcomes [24].

From literature review, M-pro seems to be a popular anti-viral drug target site now, and so many labs around the world are working on investigational and also in virtual screening research to achieve powerful and effective inhibitors. However, drug discovery is typically a trial-and-error/hit-and-miss endeavour, owing in large part to fundamental deficiencies in the fundamental understanding of the molecular and cellular structure-free energy relationships, and also dependence on equilibrium potency metrics (e.g., $\mathrm{IC}_{50}, \mathrm{~K}_{\mathrm{d}}$ ) that are constrained in their validity to non-equilibrium situations in vivo $[25,26]$. Druggable proteins, such as M-Pro, that participate in the early stages of infection prior to or during the replication construction phase, are ideal targets for therapeutic 
intervention. Clinical anti-viral success complex and virion production at a threshold fractional inhibition of the protein population over time, which may be relatively high given how each available enzyme duplicate, could even correct numerous membrane copies. As previously demonstrated, when the rates of drug association and dissociation are tuned to the rates of target or binding site build-up and decay, efficient and effective vibrant tenancy under pro circumstances has been accomplished only at least potential exposure.

A class of virulence factors is one of the body's rapid response immune response and a possible threat for anti-COVID-19 therapies. When the receptors recognise a foreign pattern, they become activated, causing the immune system to enter antiviral mode. Several researchers have been racing to understand the virus's peculiar nature and the pathogenesis of the disease in order to identify potential drug targets. Because of bacterial and viral resistance to currently available antibiotics, there is a growing interest in developing new drugs with improved activity. Because metals and ligands interact with different stages of the pathogen life cycle, they can be used to create new drugs. As a result, our current investigation has identified a number of drug targets. The C-N moiety present in Phene ligand will play an important role in terms of biological activity. So, for a number of transition and inner transition metal complexes with various biological activities such as antimicrobial, anticancer, and antifungal by using a variety of $\mathrm{N}$ - donor ligands were studied. Monitoring molecular docking levels in antiviral, antibacterial, anticancer, and antimicrobial activities is therefore a promising target therapy for evaluating response to standard COVID-19 treatments. Tetra-1,10-Phenantroline Dy(III) complex were selected to see their potential in antimicrobial and anticancer activity. We incorporate traditional screening and structure-based drug design approaches throughout this work to investigate M-pro inhibition from a theoretical, spectral based, and insilico-relevant perspective based on simple principles.

\section{Materials and Methods}

\subsection{Materials}

Dysprosium (III) trichloride hexa hydrate, BSA, Celite, Silicagel, and 1,10 phenanthroline were purchased from sigma Aldrich and used as received. Chloroform, DMF, Toluene, acetonitrile, and ethanol were purchased in Merck AR grade and used as received. Double distilled water was obtained by distilling distilled water over alkaline potassium permanganate. Diethyl ether and acetonitrile (AR, Merck) were used as received.

\subsection{Methods}

CHN microanalyses were carried out using a Perkin-Elmer 2400 Series II CHNS/O Elemental Analyzer, interfaced with a Perkin-Elmer AD 6 Autobalance. Helium was used as the carrier gas. Uv-Visible Absorption Spectrum were recorded in the 200-900 $\mathrm{nm}$ regions on Deep vision UV/VIS spectrophotometer using cuvette with a $1 \mathrm{~cm}$ path length. The concentration of ligand and metal complexes was kept at $1.00 \times 10^{-5} \mathrm{~mol} \mathrm{~L}^{-1}$, at $310 \mathrm{~K}$.

2.2.1. Bovin serum albumin binding studies: The complexes' absorption properties in the presence of BSA were determined by treating the complexes with 4.5 percent BSA in water until equilibrium was reached and then measuring the absorption over time intervals ranging from 15 minutes to 3 days. All absorption measurements were taken in a UV-Visible spectrometer and recorded at $300 \mathrm{~K}$. 
2.2.2. Molecular docking study: The docking studies were performed as described in our previous publications $[6-9,27]$.

\subsection{Experiment Methods}

2.3.1. Synthesis of Tetra-1,10-Phenanthroline Dysprosium complex [Dy(III)(Phen $\left.)_{4}\right]:$ About $0.79 \mathrm{~g}(4 \mathrm{mmol})$ of 1,10-Phenanthroline and $0.28 \mathrm{~g} \mathrm{(1} \mathrm{mmol)} \mathrm{DyCl}_{2} \cdot 6 \mathrm{H}_{2} \mathrm{O}$ in $50 \mathrm{~mL}$ of double distilled $\mathrm{H}_{2} \mathrm{O}$ and little Acetonitrile were taken in $250 \mathrm{ml} \mathrm{RB}$ flask and heated at $120^{\circ} \mathrm{C}$ under stirring for 24 hours. After the formation of pink colour solution, the mixture was cooled to room temperature. The obtained clear pink solution was evaporated to dryness under reduced pressure (Scheme-1). The pink [Dy(III)(Phen)4] complex obtained was air dried and recrystallized from water, yield $5.17 \mathrm{~g}(99 \%), \mathrm{mp} 264{ }^{\circ} \mathrm{C}$ (dec.). CHNS/O. calcd. \% for $\mathrm{C}_{48} \mathrm{H}_{32} \mathrm{DyN}_{8}(\mathrm{Mr}=883.34): \mathrm{C}, 65.27 \%$; H, $3.65 \%$; N, $12.69 \%$ : Dy, $18.40 \%$. Found C, $65.17 \%$; H, $3.45 \%$; N, $12.52 \%$ : Dy, $18.18 \%$.

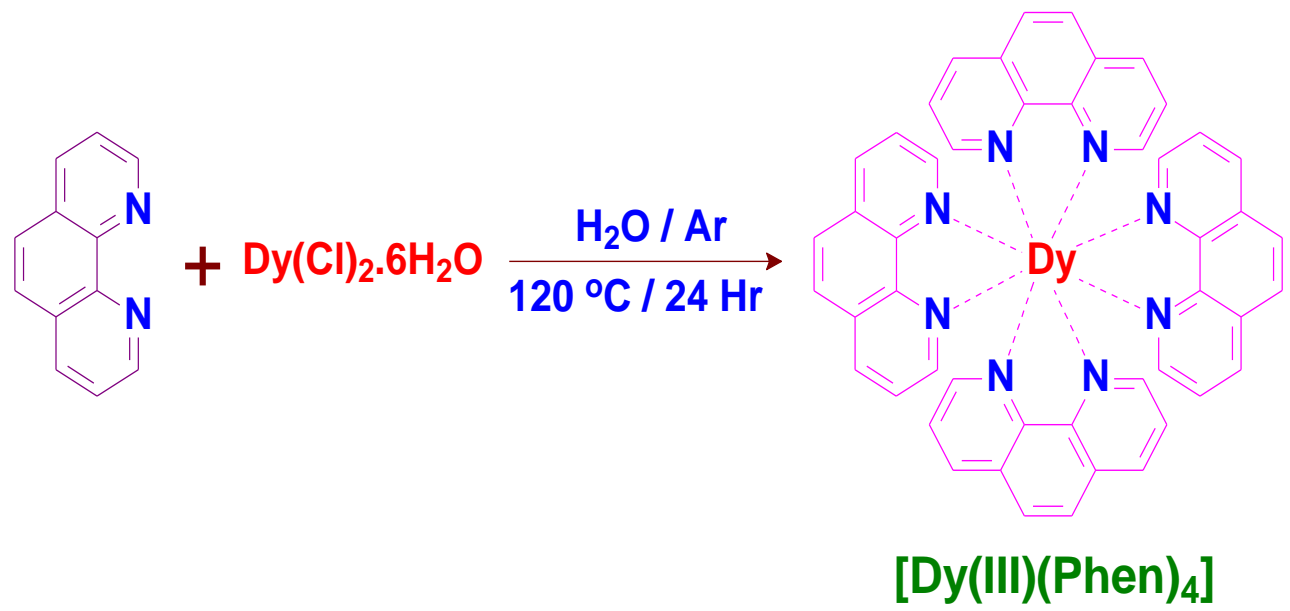

Scheme 1. Synthesis of tetrakis-1,10-phenanthroline-Dy(III) complex

\section{Result and Discussions}

\subsection{Theoretical Chemistry}

A molecule can possess different kinds of energy such as bond and thermal energy. Through insilico Molecular mechanics and by using Gaussian algorithm the steric energy and the potential energy of the complex were analyzed.

3.1.1. Steric Energy Calculation: The steric energy of a molecule is calculated using molecular mechanics as a result of its geometry or morphology. Energy is minimized in nature, and the preferred conformation of a molecule is the lowest energy conformation.

The verification of a molecule is important because the structure of a compound frequently has a large effect on its reactivity. Molecular mechanics assumes that even the stereo chemical energy of a molecule is the result of a few, direct interactions within the molecule.

These interactions include the stretching or compressing of bonds beyond their equilibrium lengths and angles, the torsional effects of twisting on single bonds, the Van der Waals attractions or points of interest with atoms that are even close together, and the electrostatic forces between partial charges in a molecule due to polar bonds. These 
interactions could be quantified by modelling them with a potential function that provides the same energy as a function of distance, angle, or charge [28,29]. The total steric energy of a molecule can be expressed as the sum of the energies of the interactions:

$E_{\text {steric energy }}=E_{\text {str }}+E_{\text {bend }}+E_{\text {str-bend }}+E_{\text {oop }}+E_{\text {tor }}+E_{\mathrm{VdW}}+E_{q q}$

Entwined encounters involve bond deformation, bending, stretch-bend, out of plane, and torsion because the atoms involved must be directly bonded or bonded to something similar to a common atom. Van der Waals and electrostatic (qq) interactions exist between non-bonded atoms. Table 1 displays the complex's steric energy.

3.1.2. Potential energy calculation: Potential energy is the distinction in energy between an object's energy in one position and its energy in another. Potential energy is frequently linked to restoring forces like a spring or gravity.

Table 1. Calculated minimized energies for $\left[\mathrm{Dy}(\mathrm{III})(\mathrm{Phen})_{4}\right]$ complex molecule

\begin{tabular}{|c|c|c|c|}
\hline Si. No. & Calculated Values & \multicolumn{2}{|c|}{$\left[\mathrm{Dy}(\mathrm{III})(\text { Phen })_{4}\right]$} \\
\hline 1. & Stretch & & 4.4662 \\
\hline 2. & Bend & & 10.0661 \\
\hline 3. & Stretch-Bend & & 1.4529 \\
\hline 4. & Torsion & & -8.2845 \\
\hline 5. & Non-1.4 VDW & & -8.9412 \\
\hline 6. & $1.4 \mathrm{VDW}$ & & 35.5582 \\
\hline 7. & Dipole Dipole & & 1.3664 \\
\hline 8. & Total Energy & 35.6840 & Kcal/mol \\
\hline 9. & Potential Energy & 35.665 & \pm 0.095 \\
\hline 10. & Steric Energy & 349: $35.684 \mathrm{k}$ & $\mathrm{cal} / \mathrm{mole}$ \\
\hline
\end{tabular}

External force acting against the potential's force field performs the action of stretching this same spring or lifting the mass of the object. This work is stored with in force field as energy potential. When the external force is removed, the force field acts on the body to perform the tasks by returning it to its initial position, decreasing the extension of the spring, or starting to cause the body to fall. The much more proper definition would be that potential energy is indeed the energy difference between an object's energy inside one position and its energy in the other. Table-1 shows the complex's potential energy.

\subsection{Electronic Absorption Studies}

3.2.1. Absorption spectrum of complex: The UV-Vis absorbance spectra of the [Dy(III)(Phen)4] complex in water is presented in figure 1. In the short wavelengths region of the spectrum the spectral curve has a broad maxima with multiple less intense peak corresponding to the transitions from the ground ${ }^{4} \mathrm{M}_{19 / 2},{ }^{6} \mathrm{P}_{3 / 2},{ }^{6} \mathrm{P}_{5 / 2},{ }^{4} \mathrm{I}_{11 / 2},{ }^{4} \mathrm{M}_{15 / 2},{ }^{6} \mathrm{P}_{7 / 2}(300$ to $397 \mathrm{~nm}$ ). There are several closely situated energy levels around $250-300 \mathrm{~nm}$, i.e., ${ }^{4} \mathrm{~F}_{5 / 2},{ }^{4} \mathrm{I}_{9 / 2},{ }^{4} \mathrm{G}_{9 / 2},{ }^{4} \mathrm{M}_{17 / 2},{ }^{6} \mathrm{P}_{3 / 2}$, 
${ }^{4} \mathrm{~K}_{15 / 2},{ }^{4} \mathrm{~L}_{19 / 2},{ }^{4} \mathrm{G}_{7 / 2},{ }^{4} \mathrm{D}_{5 / 2},{ }^{4} \mathrm{D}_{1 / 2}$. Transitions to all the levels would be of very low intensity.

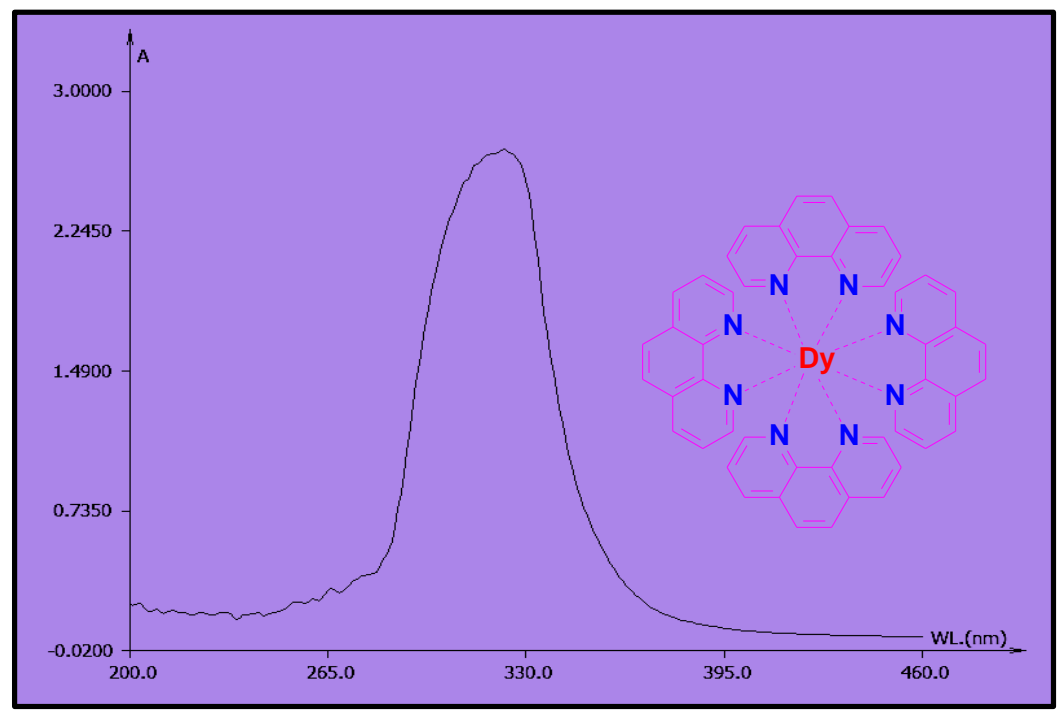

Fig.1. Electronic absorption spectrum of $\left[\mathrm{Dy}(\mathrm{III})(\mathrm{Phen})_{4}\right]$ complex in water

3.2.2. Absorption spectrum of complex with BSA: The UV-Vis absorbance spectrum of the complex in water along with $4.5 \%$ of BSA is presented in figure 2. In the UV-region, the complex displays similar bands with respect to free metal complex. The transitions from the ground ${ }^{4} \mathrm{M}_{19 / 2} ;{ }^{6} \mathrm{P}_{3 / 2},{ }^{6} \mathrm{P}_{5 / 2},{ }^{4} \mathrm{I}_{11 / 2}$ (between 310-418 nm); ${ }^{4} \mathrm{M}_{15 / 2}$ and ${ }^{6} \mathrm{P}_{7 / 2}(350 \mathrm{~nm})$ is obtained as like the complex in solvent alone. In the presence of BSA protein the absorption values for the complex are shifted due to the polar interaction of metal complex with BSA. The red shift in the absorption value from 323 to 318 and 266 to $260 \mathrm{~nm}$ confirms the binding interacted energy transfer like LCT and MLCT of the complex with BSA protein.

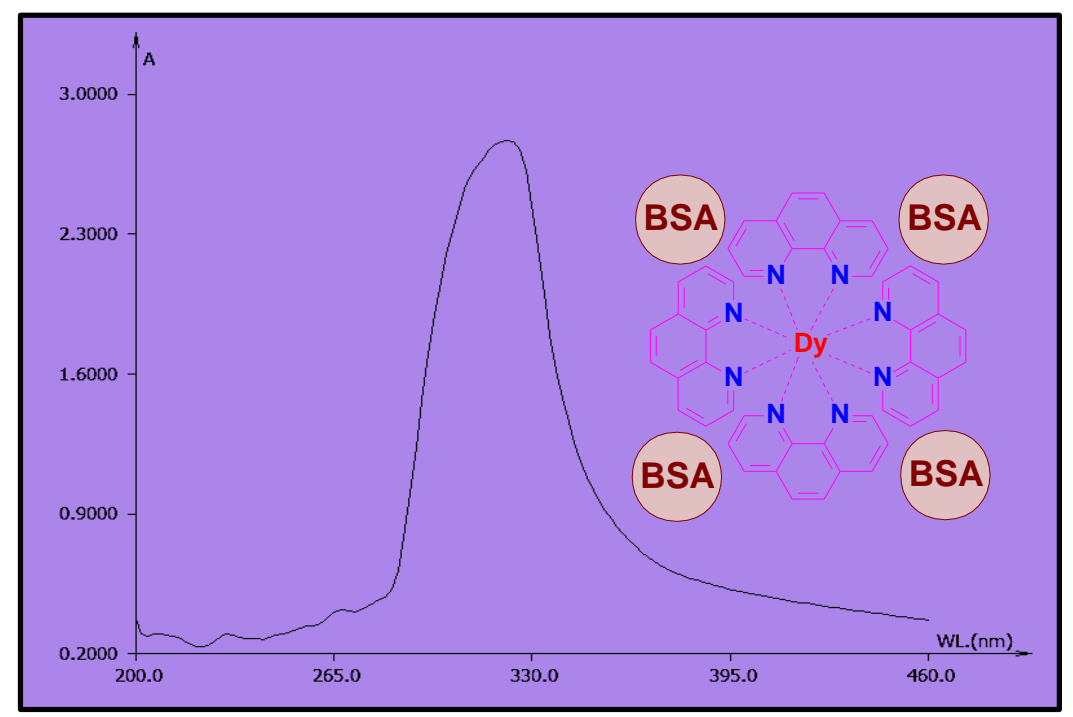

Fig.2. Electronic absorption spectrum of $\left[\mathrm{Dy}(\mathrm{III})(\mathrm{Phen})_{4}\right]$ complex with $4.5 \%$ BSA in water

\subsection{Molecular docking study with SARS-CoV-2 (PDB: 6LU7)}

The insilico molecular docking study on the complex has studied to identify the possible binding sites on the corona virus. The main protease on the spike protein 'S' contains the key amino acids ALA, ARG, ASN, ASP, CYS, GLN, GLU, GLY, HIS, ILE, LEU, LYS, MET, PHE, PRO, SER, THR, TYR, and VAL which makes the molecule high polar 
and support in binding with human ACE-2 enzyme for multiplication. Also these amino acids are the main source of energy providers, replication initiator, and salt bridge former between S1 and S2 to keep the virus anatomy stable. The binding efficiency of our [Dy(III)(Phen)4] complex with the virus amino acid sequence were performed and their binding site and the depth of burying has been calculated. As it is expect the highly magnetic Dy(III) complex strongly anchors through $\pi$ - interaction with key amino acids like, ALA-285, ASP-153, 197, 245, ILE-106, LEU-286, LYS-5, 137, 236, PHE-294, THR-198 and TYR-154 in COVID-19 spike protein with a coupling energy of $-8.8 \mathrm{kcal} \mathrm{mol}^{-1}$. The docking structure confirms that our complex strongly binds with the M-Protease of the COVID-19 spike protein and gives information about the change in virus enzymatic action during replication. The polar phenanthroline ligand can be functionalized with other antivirus drug and can be used to treat covid-19 infections. The binding sites and their orientations are given in figure 3.
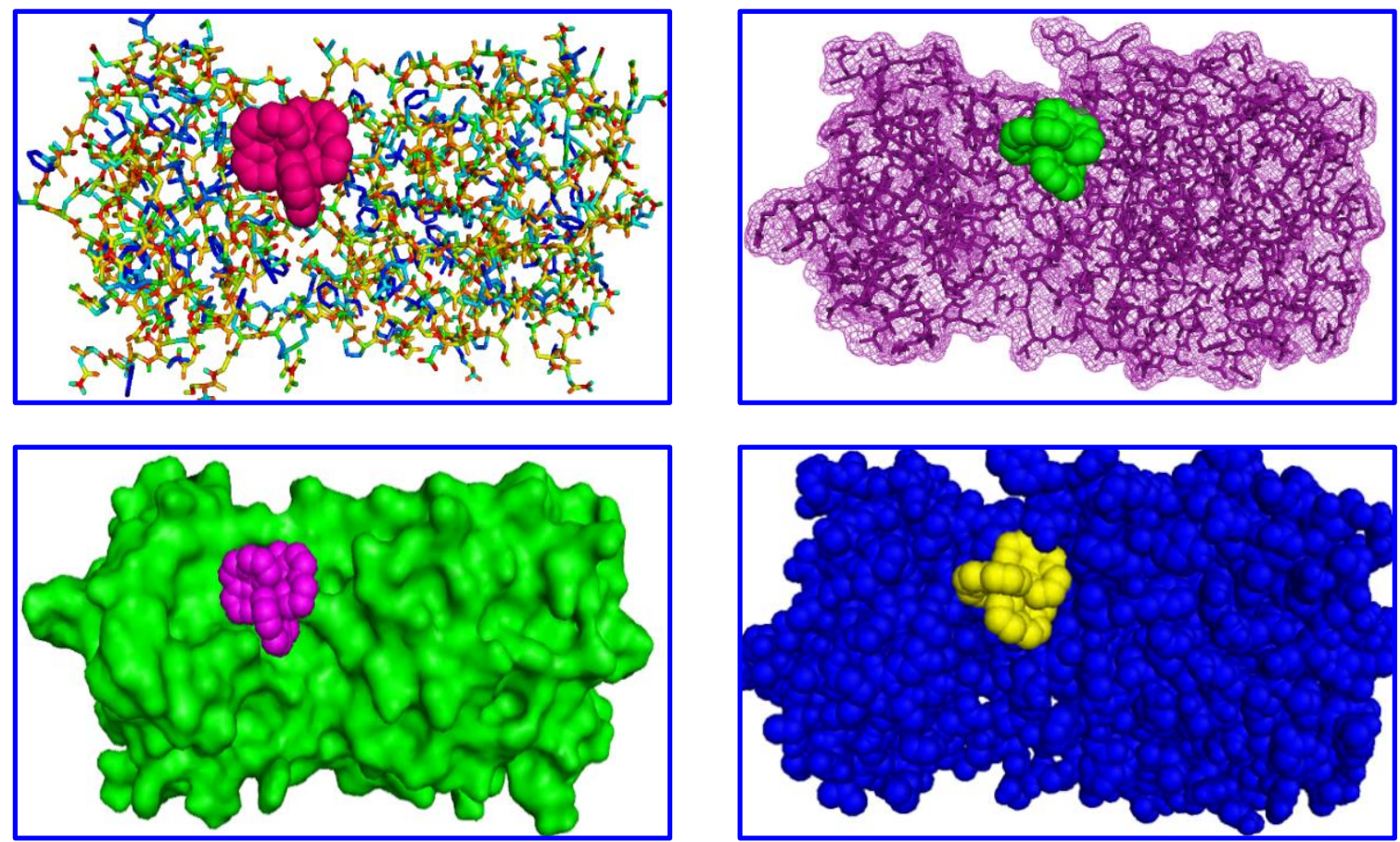

Fig.3. Molecular Docked Images of [Dy(III)(Phen)4] complex with COVID-19 spike Protein (PDB No.: 6LU7)

\section{Conclusion}

The $N$ - donor heterocyclic ligand, 1,10-phenanthroline, based Dy(III) metal complex has been reported to inhibit the covid-19 replication process in human. The binding efficiency was evaluated by Invitro and insilico methods. Initially the binding affinity with simple serum albumin was done using Uv-Visible spectroscopy. In the presence of $4.5 \%$ BSA the complex shows red shift in absorption value due to the polar interactions and the Macrocyclic effect of the BSA molecule. This binding affinity examination was extended with insilico method on SARS-CoV-2 3D crystal structure. The molecular docking study confirms the binding capability of our complex over covid-19 main protease which is responsible for the binding on ACE-2 in human. Also the higher binding energy (-8.8 $\mathrm{kcal} / \mathrm{mol}$ ), steric and kinetic inertness confirms the suitability of the smart Dy(III) complex to behave as drug for anti-covid studies. Soon the complex will be recognized as a potential antiviral agent for other microbes responsible for Covid like infections. 


\section{Declarations}

\section{Source of Funding}

This research did not receive any grant from funding agencies in the public, commercial, or not-for-profit sectors.

\section{Competing Interests Statement}

The authors declare no competing financial, professional and personal interests.

\section{Ethical Approval}

Ethical approval for this research was given based on institutional guidelines.

\section{Consent to participate}

The consent to participate in this research was sought for and approved by the subjects to be used.

\section{Consent for publication}

We declare that we consented for the publication of this research work.

\section{Availability of data and material}

Authors are willing to share data and material according to the relevant needs.

\section{References}

[1] Bui, A. T. et al. Near infrared two photon imaging using a bright cationic $\mathrm{Yb}(\mathrm{III})$ bioprobe spontaneously internalized into live cells. Chem. Commun. 2017, 53, 6005-6008.

[2] Yan, B. Lanthanide-functionalized metal-organic framework hybrid systems to create multiple luminescent centers for chemical sensing. Acc. Chem. Res. 2017, 50, 2789-2798.

[3] Hasegawa, Y. \& Kitagawa, Y. Thermo-sensitive luminescence of lanthanide complexes, clusters, coordination polymers and metal-organic frameworks with organic photosensitizers. J. Mater. Chem. 2019, C 7, 7494-7511.

[4] T.Maier, H.Kadau, M.Schmitt, M.Wenzel, I.Ferrier-Barbut, T.Pfau, A.Frisch, S.Baier, K.Aikawa, L.Chomaz, M.J.Mark, F.Ferlaino, C.Makrides, E.Tiesinga, A.Petrov, S.Kotochigova, Emergence of chaotic scattering in ultracold Er and Dy. Phys. Rev. X 2015, 5, 041029.

[5] A. Frisch, M. Mark, K. Aikawa, F. Ferlaino, J. L. Bohn, C. Makrides, A. Petrov, S. Kotochigova, Quantum chaos in ultracold collisions of gas-phase erbium atoms. Nature, 2014, 507, 475-479.

[6] T.Lurthu Pushparaj, M.Uma Devi, 'Developing Novel Coumarone-Phenyl Amide Functionalized [Gd(III)-Pt (IV)] Complex as High T1, T2 Relaxive M-MRI Contrast Agent for Cancer Diagnosis, IIJSR, 2021, 5, 51-63.

[7] T.Lurthu Pushparaj, E.Francy Irudaya Rani, M.Uma Devi, 'HSA and CA-125 Binding Study of [Pr-(DO3 -Ch-Ph-Am-Gd(III))2Pt(IV)] Complex as M-MRI Contrast Agent for Ovarian Cancer Treatment', JIDPTS, Volume 4, Pages 1-8, 2021. 
Asian Journal of Applied Science and Technology (AJAST)

Volume 5, Issue 2, Pages 124-133, April-June 2021

[8] T.Lurthu Pushparaj, E.Francy Irudaya Rani, S.Sathya, 'Therapeutic Properties of Gd(III)-Ir(III) Complex for Non-invasive Detection of Ovarian Cancer Through M-MR Imaging', Irish Interdisciplinary Journal of Science \& Research, 2021, 5, 23-33.

[9] T.Lurthu Pushparaj, S.Sathya, 'Novel Designing of Ir(III)(Tris-Coumarin) Cored Gd(III) Complex as Targeted MRI CAs for Ovarian Cancer Treatment', Irish Interdisciplinary Journal of Science \& Research, 2021, 5, 7-17

[10] T.Lurthu Pushparaj, V.Alexander, 'High Rigid Gd(DO3VA) Shows Remarkable Relaxivity: A Novel Class of MMI Agent Engineered for MR Analysis’, App.Chem. Eng., 2018, 1, 762.

[11] T.Lurthu Pushparaj, V.Alexander, 'Trinuclear Gd(III) Metal Complex with Amide Core Display Remarkable Enhancement in Relaxivity’, Appl. Magn. Reson., 2017, 48, 813-825.

[12] T.Lurthu Pushparaj, V.Alexander "Synthesis, pH and HSA Binding study of Novel Dinuclear [Gd(III)DO3 VA] complex as Magnetic Resonance Imaging Contrast agent” Int. J. Scientific. \& Engg. Res., 2016, 7, 1600-1605.

[13] T.Lurthu Pushparaj, V.Alexander, "Development of Novel Dinuclear [Gd(III)DO3VA] Complexes Decorated with Isovaleric acid as MRI Contrast Agents for Tumor Diagnosis”, Int. J. Appl. Bio-engg., 2016, 10, 11-17.

[14] T.Lurthu Pushparaj, V.Alexander, "Synthesis and Relaxivity Measurements of Novel Gd(III) Complex of DOTVA as MRA Contrast Agents", Int. J. Appl. Bio-engg., 2014, 8, 1-8.

[15] Luman, C.R., Castellano, F.N., "Phenanthroline Ligands" in Comp. Coord. Chemistry II, Elsevier, 2003.

[16] Sammes Peter G., Yahioglu Gokhan, "1,10-Phenanthroline: A versatile ligand", Chem. Soc. Rev., 23, 1994.

[17] Felber, Jean-Pierre, Coombs, Thomas L., Vallee, Bert L., "The mechanism of inhibition of carboxypeptidase A by 1,10-phenanthroline", Biochemistry, 1962, 1, 231-238.

[18] Salvesen, G.S., Nagase, H., "Inhibition of proteolytic enzymes", In Beynon, Rob \& Bond, J S (eds.), Proteolytic Enzymes: A Practical Approach, 1 (2nd ed.), Oxford University Press, 2001, pp.105-130.

[19] Arunkumar, T., Sharanappa, B., Nandibewoor, T., "Multi-spectroscopic characterization of bovine serum albumin upon interaction with atomoxetine", J. Pharmaceutical Analysis, 2016,7(3), 148-155.

[20] Guan, Y., et al., "Isolation and characterization of viruses related to the SARS coronavirus from animals in southern China", Science, 2003, 302, 276-278.

[21] Arabi, Y. M., et al., “Middle East Respiratory Syndrome”, The New Eng. J. of Med., 2017, 376 (6), 584-594.

[22] Kim, J.-M., et al., "Identification of coronavirus isolated from a patient in Korea", Osong Public Health Res Perspect, 2020, 11 (1), 3-7.

[23] Manjili, R. H., Zarei, M., Habibi, M. \& Manjili, M. H., "COVID-19 as an acute Iinflammatory dsease”, The Journal of Immunology, 2020, 2000413.

[24] Ackermann, M. et al., "Pulmonary vascular endothelialitis, thrombosis, and angiogenesis in covid-19", The New England Journal of Medicine, 2020, 383, 120-128. 
[25] Selvaggio G, Pearlstein RA., "Biodynamics: A novel quasi-first principles theory on the fundamental mechanisms of cellular function/dysfunction and the pharmacological modulation thereof", PLoS One, 13, 2018.

[26] Pearlstein RA, McKay DJJ, Hornak V, Dickson C, Golosov A, Harrison T., "Building New Bridges between In Vitro and In Vivo in Early Drug Discovery: Where Molecular Modeling Meets Systems Biology", Curr Top Med Chem., 17: 1-1, 2017.

[27] Trott, O., Olson, A. J., "Auto Dock Vina: improving the speed and accuracy of docking with a new scoring function, efficient optimization and multithreading”, J. Comp. Chem., 31, 455-461, 2010.

[28] Kuntz, I. D., Thomason, J. F., Oshiro, C. M., “Distance Geometry” in Methods in Enzymology, Volume 177 Nuclear Magnetic Resonance Part B, Oppenheimer, N. J., James, T. L., Eds., Academic Press: San Diego, CA, 1989, pp. 159-204.

[29] Crippen, G. M., Havel, T. F., "Distance Geometry and Molecular Conformation", Bawden, D., Ed., Chemometrics Research Studies Series 139, Research Studies Press (Wiley): New York, 1988. 